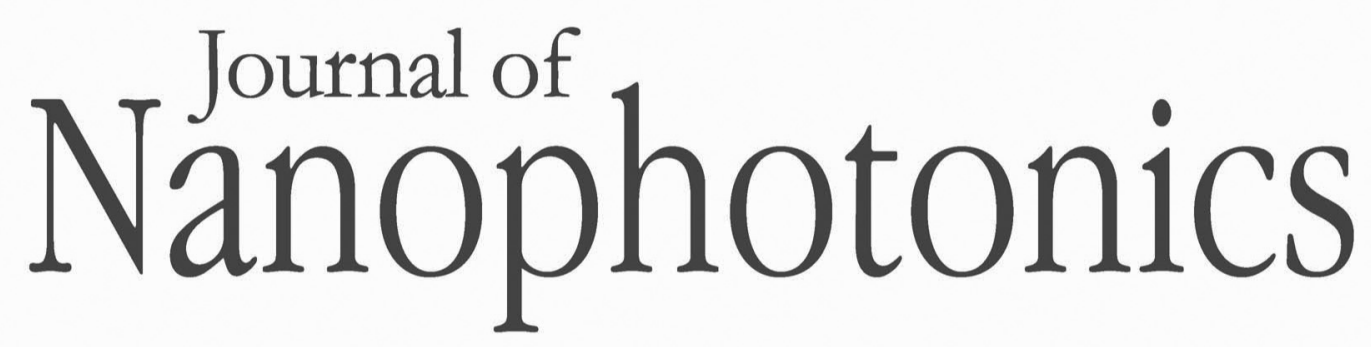

\title{
High birefringence triangular optical nanowire in suspended-core fiber for temperature sensing
}

Ricardo M. André

Martin Becker

Kay Schuster

Manfred Rothhardt

Hartmut Bartelt

Manuel B. Marques

Orlando Frazão 


\title{
High birefringence triangular optical nanowire in suspended-core fiber for temperature sensing
}

\author{
Ricardo M. André, ${ }^{\text {a,b }}$ Martin Becker, ${ }^{\mathrm{c}}$ Kay Schuster, ${ }^{\mathrm{c}}$ Manfred Rothhardt, ${ }^{\mathrm{c}}$ \\ Hartmut Bartelt, ${ }^{\mathrm{c}}$ Manuel B. Marques, ${ }^{\mathrm{a}, \mathrm{b}}$ and Orlando Frazão ${ }^{\mathrm{a}}$ \\ ${ }^{a}$ Instituto de Engenharia de Sistemas e Computadores do Porto, Rua do Campo Alegre 687, \\ 4169-007 Porto, Portugal \\ randre@inescporto.pt \\ ${ }^{b}$ University of Porto, Physics and Astronomy Department, Faculty of Science, Rua do Campo \\ Alegre 687, 4169-007 Porto, Portugal \\ 'IPHT, Institute for Photonic Technology, D-07745 Jena, Germany
}

\begin{abstract}
Triangular nanowires that present a high birefringence and a very strong confinement were fabricated by tapering suspended-core fibers (SCFs) down to core diameters below $1000 \mathrm{~nm}$. Each nanowire presented a high birefringence with an order of magnitude of $10^{-3}$. As the spectra of the SCF tapers inserted in fiber loop mirrors can be used to generate a sinusoidal interference pattern from the two main modes (fast and slow axis), a nanowire was employed as a sensing element in a Sagnac interferometer for measuring temperature. Temperature sensitivity was determined to be $-56.2 \mathrm{pm} / \mathrm{K}$ using a triangular nanowire of $810 \mathrm{~nm}$ in-circle diameter when compared with that of a conventional untapered SCF whose temperature sensitivity is $-2.1 \mathrm{pm} / \mathrm{K}$. (c) 2013 Society of Photo-Optical Instrumentation Engineers (SPIE) [DOI: 10.1117/1.JNP.7.073088]
\end{abstract}

Keywords: optical fiber nanowires; Sagnac interferometer; optical fiber sensors; suspended-core optical fiber; high birefringence.

Paper 13014 received Feb. 21, 2013; revised manuscript received Jun. 3, 2013; accepted for publication Jun. 11, 2013; published online Jul. 8, 2013.

\section{Introduction}

Optical fibers based on silica with light-guiding transverse dimensions larger than the wavelength of light have become an irreplaceable asset in optical communications, sensing, power delivery, and nonlinear optics. As for sensing, optical fiber sensors present several advantages when compared with conventional sensors: they are ideal for remote sensing and they are immune to electromagnetic noise and can be used in extreme environments where explosive gases are present. Conventional electric sensors are susceptible to interference from strong electric and magnetic fields and cannot be used in explosive gas environments as they could easily ignite the atmosphere during normal operation.

Optical fibers have been used in the most diverse fields such as overseas communication, laser surgery, and astronomical research. Recent advances in nanotechnology have led to an increasing demand for devices with faster responses, higher sensitivities, lower power consumption, and smaller dimensions. Conventional optical fibers are usually considered to be small components of devices, but when compared with nanoscale components, they become quite large devices. This resulted in the miniaturization of optical fibers and fiber-optic devices and has been the main motivation to produce optical fiber nanowires. Optical fiber nanowires are subwavelength waveguides that confine light to dimensions smaller than its wavelength. They allow for smaller dimension devices with faster responses and higher sensitivities and combine easily with integrated optics at the micro- and nanometer scale.

Most optical fiber nanowires have been fabricated via tapering of fibers with standard outer diameter greater than $100 \mu \mathrm{m} .{ }^{1-4}$ For these fibers, the whole cross-section acts as a fiber core and

0091-3286/2013/\$25.00 () 2013 SPIE 
guides light. These nanowires are fully exposed to external contamination and degradation. ${ }^{3}$ This degradation has been prevented by embedding the wires in different types of materials such as Teflon. ${ }^{5}$ The problem with this approach is that the whole structure loses its flexibility and ultimately limits its applicability. An alternative solution to the problem is to use suspended-core fiber (SCF). ${ }^{6}$ One of the main advantages of SCFs is the fact that light is well confined to the core, when it is large, because of the high step index profile of the silica-air interface between core and air holes. When the core is small, a large evanescent field exists but is nonetheless contained within the fiber, especially the fiber's holes. This allows for a very high protection from the external medium and no interaction with the surrounding medium's refractive index occurs. ${ }^{7}$ It was shown that microstructured optical fibers can be tapered while retaining their cross-sectional profile with no distortion. ${ }^{8,9}$ The cross-sectional profile can thus be scaled down proportionally, producing structures with very small core diameters. ${ }^{10}$ The SCFs have already been produced with very small suspended cores. ${ }^{11}$ Two approaches for fabricating SCFs with very small cores have been employed: direct drawing of long length SCFs with subwavelength core sizes and practical outer diameters larger than $100 \mu \mathrm{m}^{12}$ and tapering of SCFs. ${ }^{13}$

In this article, suspended-nanocore fiber tapers in Sagnac interferometer configurations are studied. After an initial modal characterization of the core geometry, analyses of the group birefringence of the suspended nanowires and their temperature sensitivities are performed.

\section{Fabrication and Simulation}

The combination of the core size with the large step index difference from silica to air leads to the highly multimode nature of SCF. The advantage of large-core SCFs is the easy coupling to standard single-mode fibers. On the other hand, the advantage of small-core SCFs is that they guide a small number of modes and present a cleaner spectral response. A compromise in size is then necessary to assure that the SCF is relatively easy to connect to standard fiber and that it does not guide too many modes.

A solution is to start with an SCF with a $2.1-\mu \mathrm{m}$ core. An SCF with a core this size already has few modes and is easy to splice with a standard monomode fiber. The splices are made manually with a conventional electric arc discharge splicing machine. ${ }^{14}$ To splice SCF and standard monomode fiber with small losses and without collapsing the air holes, it is necessary to lower the arc current, shorten the arc duration, and shift the arc to the standard monomode fiber section. When tapering the SCF and analyzing its spectral response, an interference pattern between a smaller number of modes is visible. At the down-taper region, some guided modes are coupled to radiation modes and their energy is radiated and lost along the taper. This is the reason why the resultant interference pattern of a tapered SCF reveals fewer modes than an untapered one. The main idea is to taper the SCF reducing the core diameter below $1 \mu \mathrm{m}$. This will lead to a completely different spectral response and enhanced sensitivity to physical parameters. The enhancement is expected due to the modification of the mechanical and optical properties generated by the fabrication of the taper.

The SCF used has an external diameter of $125.6 \mu \mathrm{m}$ and a core diameter of $2.1 \mu \mathrm{m}$ [Fig. 1(a) and 1(b)]. In the core, a small germanium-doped region with a diameter of $0.7 \mu \mathrm{m}$ also exists. The air holes are roughly elliptical $\left(10.7 \times 14.3 \mu \mathrm{m}^{2}\right)$. The thin bridges that connect core to cladding have widths that range from 170 to $230 \mathrm{~nm}$. The triangular shaped core presents a high asymmetry and, consequently, a high birefringence.

\subsection{Taper Production}

The tapers were produced using a VYTRAN Glass Processing Workstation. The VYTRAN is a glass processing platform that performs fusion splicing and tapering of specialty fibers. The system consists of a filament heater, precision stages with multiaxis control (fiber-holding blocks), a microscopic high resolution charge-coupled device imaging system, and a personal computer. The filament heater has a wide temperature range from several hundreds up to $3000^{\circ} \mathrm{C}$, which allows the easy tapering of microstructured optical fibers. The working principle is based on heating a portion of fiber in the filament heater to its softening point while applying a tensile 


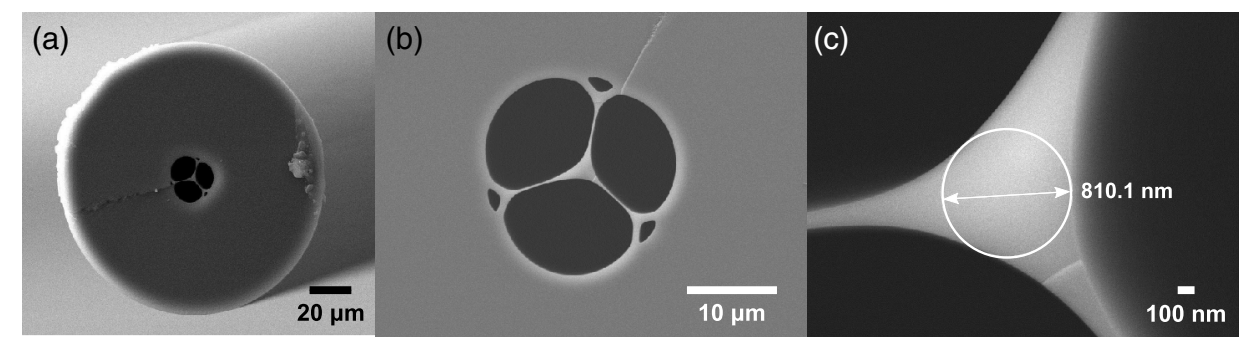

Fig. 1 SEM micrographs: (a) cross-section of the $125 \mu \mathrm{m}$ untapered fiber; (b) $2.1 \mu \mathrm{m}$ core; and (c) $810 \mathrm{~nm}$ core.

force by pulling the fiber with the holding blocks. As the fiber elongates, its cross-sectional area will be reduced accordingly. The whole tapering process takes less than $30 \mathrm{~s}$ for any taper diameter considered. With the VYTRAN, it is possible to taper the fiber down to $330 \mathrm{~nm}$ of core diameter (20- $\mu$ m cladding diameter) while maintaining the cross-section geometry; this is an effective reduction to $16 \%$ of the original size (see Fig. 2). The VYTRAN effectively eliminates the risk inherent to tapering a microstructured optical fiber with air holes. It reduces the fiber diameter by scaling the cross-section, maintaining the geometry and avoiding deformation and hole collapse.

The main parameters that can be adjusted when tapering the fibers with the VYTRAN are the taper pull velocity and the filament power (direct control over filament temperature). After the intended down taper, taper waist and up-taper lengths are decided upon (in this case 15-10$15 \mathrm{~mm}$ ) and the taper waist diameter is set, the range of taper pull velocity and filament power is restricted. These two parameters are controlled so that the tension the fiber is subjected to during taper fabrication is neither too high nor too low. Too low filament temperature will lead to low viscosity, high tension, and the fiber will break. Too high filament temperature will lead to high viscosity, low tension and slack will be created leading to an asymmetric taper relative to the fiber axis. At high temperatures, hole collapse ensues at higher taper waist. As for repeatability, several tapers with the same characteristics were produced using the same parameters. All this means that the parameters are pretty well established once the structure we want to obtain is defined.

\subsection{Simulations}

The number of modes guided by this specific suspended-core structure was studied using the beam propagation method. Clearly seen is that the number of modes, although discrete, increases quadratically with increasing core diameter [see Fig. 3(a)]. In the suspended core, the core

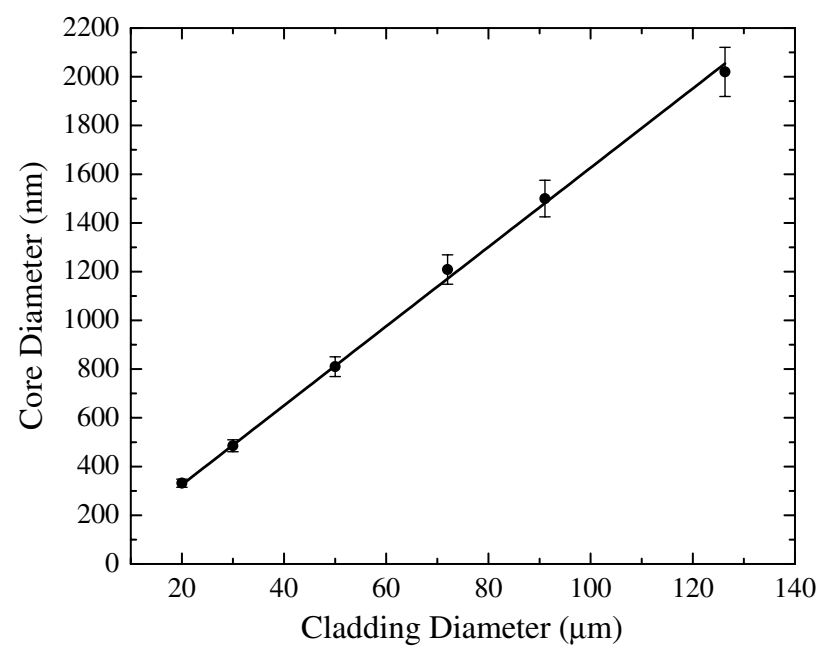

Fig. 2 Linear relationship between core diameter and cladding diameter. 

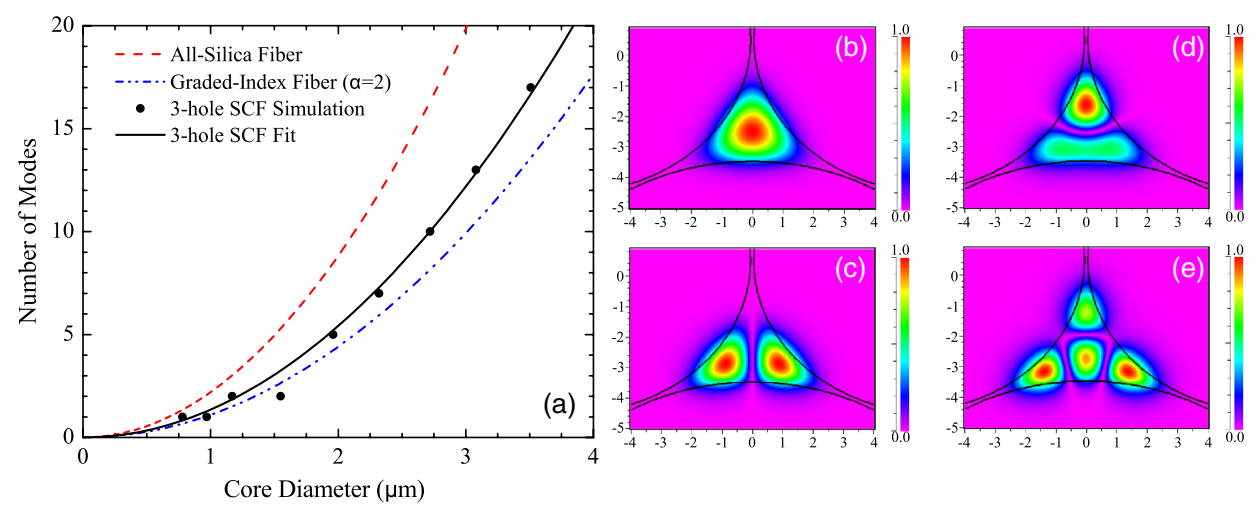

Fig. 3 (a) Quadratic increase of the number of guided modes with the core diameter; (b-e) electric field amplitude of the first four guided modes in the three-hole suspended-core geometry.

diameter is taken to be the in-circle diameter of the core. An approximate expression for the number of modes as a function of the normalized frequency $\left(V=\frac{2 \pi r}{\lambda} \sqrt{n_{\text {core }}^{2}-n_{\text {clad }}^{2}}\right)$ was obtained from fitting the simulation data in Fig. 3(a) and is as follows:

$$
N \approx \frac{V^{2}}{3}
$$

Comparing this result with the expression for a step-index multimode fiber $\left(N=V^{2} / 2\right)$ and a standard graded-index fiber ( $N=V^{2} / 4$ for $\alpha=2$ ), one sees that the SCF falls somewhere in between. This is expected since the suspended core can be modeled as a graded-index fiber if one considers the effective index in circles with increasing diameter around the core center. This model would approximate the suspended core to a graded-index fiber with an $\alpha$ larger than 2, effectively placing it between a conventional graded index $(\alpha=2)$ and a step-index fiber $(\alpha=\infty)$.

In Fig. 3(b)-3(e), the electric field amplitude of the first four guided modes is represented for an untapered SCF at a radiation wavelength of $1550 \mathrm{~nm}$. Below a core diameter of $1 \mu \mathrm{m}$, the simulation indicates that only one mode is present, not taking into account the polarization state. The second mode cut-off diameter of the triangular nanowire is quite similar to the cut-off diameter of a standard silica fiber in air $(1.1 \mu \mathrm{m})$ when considering an operating wavelength of $1.55 \mu \mathrm{m}$. A large difference is not expected since in both cases the same index difference is considered. This means that below $1 \mu \mathrm{m}$ approximately, and due to the fiber's intrinsic birefringence, only two modes will be guided.

These simulations show an important size-dependent characteristic of these tapered structures: the number of modes reduces drastically when decreasing the core diameter until only two modes survive: the fast and slow modes of the birefringent core.

\section{Results}

The tapered SCF sections $(L=50 \mathrm{~mm})$ spliced between single-mode fibers were introduced in a Sagnac interferometer. The interferometer is formed by a splice between the output ports of an optical coupler (see Fig. 4). If a birefringent fiber section was not introduced in the loop, the two beams (clockwise and counterclockwise) would travel inside the loop but having traced the same optical paths, they would interfere constructively at the coupler. In this way, all the light would be reflected back into the input port with reflectivity limited only by losses in the fiber, the splices, and the coupler. With the introduction of a section of highly birefringent fiber in the loop, the beams will be decomposed in two others after they traverse the high birefringence fiber section, corresponding to the slow and fast axis of the fiber. After completing the loop, the counter propagating beams will recombine at the coupler and interfere according to the phase difference between them. The greatest advantage of the Sagnac interferometer is that the interference 


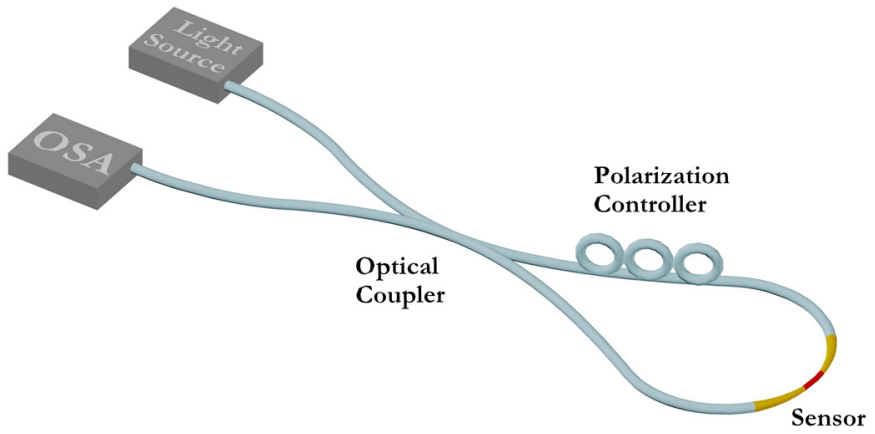

Fig. 4 Schematic representation of a Sagnac interferometer formed by a $3 \mathrm{~dB}$ optical coupler, a polarization controller, and a tapered section of suspended-core fiber. An optical broadband source and an optical spectrum analyzer are also included.

pattern is independent of the input polarization and only depends on the length of the high birefringence fiber and not the total loop length. At the input ports of the 3-dB optical coupler, a light source and an optical spectrum analyzer (OSA) were placed. The erbium-doped broadband source has a central wavelength of $1550 \mathrm{~nm}$ and a spectral width of $100 \mathrm{~nm}$. The OSA has a maximum resolution of $0.01 \mathrm{~nm}$ and is introduced to measure the output spectral response of the system. In the loop, besides the section of SCF a polarization controller was also introduced. The polarization controller allows, through rotation, the control of the angle between the polarization and the fast axis of the highly birefringent fiber and consequently allows the maximization of the visibility of the spectrum. ${ }^{15}$

It is possible to calculate the group birefringence of these tapered SCF structures from their spectrum by using the following equation: ${ }^{16}$

$$
G(\lambda)=\frac{\lambda^{2}}{\Delta \lambda \cdot L},
$$

where $\lambda$ is the operating wavelength, $\Delta \lambda$ is the fringe period, and $L$ is the length of the SCF section.

Since the SCF has a triangular core, it is expected to have a high group birefringence. In fact, the untapered fiber has a group birefringence of $3.04 \times 10^{-3}$ in the 1550 -nm region. As the fiber is tapered down to a smaller taper waist, the group birefringence increases until it reaches a maximum (see Fig. 5). It then decreases when further decreasing the diameter. The increase can be explained by the additional stresses introduced in the taper region. The decrease is likely

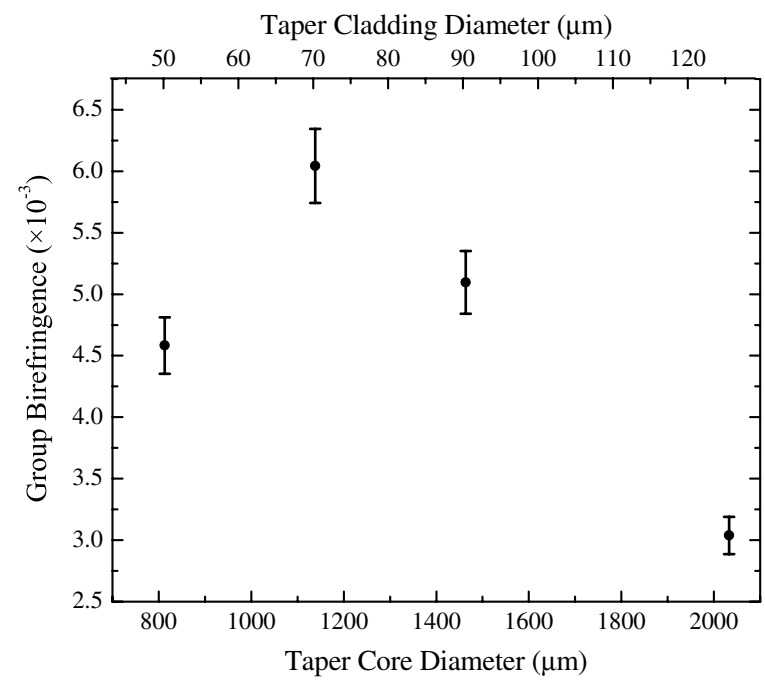

Fig. 5 Group birefringence dependence on taper diameter in the 1550-nm region. 
due to the fact that, since the wavelength of light becomes much larger than the nanocore $(1550 \mathrm{~nm}>810 \mathrm{~nm})$, the modal profile becomes so large compared with the core that it no longer depends on its geometrical shape. The superconfinement of light in the nanowire becomes weaker leading to the decreasing birefringence. The birefringence dependence on diameter allows for a new degree of control over the sensors and the construction of a sensor with a specific birefringence is possible by fabricating a simple taper.

Analyzing the spectral response of the Sagnac interferometer with the untapered SCF section [see Fig. 6(a)], one can readily observe two different interferometers: one that results from the high birefringence fiber (large-period fringes) and one that results from intermodal interference (short-period fringes). Going to smaller taper waists, the intermodal interference becomes weaker [1.5- $\mu \mathrm{m}$ core, Fig. 6(b)] and at a nanocore diameter of $810 \mathrm{~nm}$, only the group birefringence is visible and the visibility of the intermodal fringes is almost reduced to zero [see Fig. 6(c)]. This is in accordance with the previous simulations, where at $810 \mathrm{~nm}$ only two modes seem to exist. A clear advantage of tapering the suspended core down to nanometric dimensions is that the response becomes a typical spectrum from a Sagnac interferometer containing a section of conventional high birefringence fiber eliminating intermodal interference; it becomes a sinusoidal response.

The response of the untapered and tapered suspended-core structures to temperature variations in the range from $25^{\circ} \mathrm{C}$ to $75^{\circ} \mathrm{C}$ was also analyzed. For the temperature measurements, the SCF tapers were introduced in water and the spectrum was monitored. No change in the spectrum was verified when the tapers were introduced in water confirming that the sensors are insensitive to the external medium and completely protected from index changes outside the fiber. Also, during temperature measurements, no change in visibility was observed as the temperature was changed. In Fig. 7, the wavelength shift of the group birefringence fringe pattern is analyzed as a function of temperature variation for the untapered fiber and the 810-nm core taper. For the untapered case, a very low sensitivity of $-2.1 \mathrm{pm} / \mathrm{K}$ is determined, but when considering the $810-\mathrm{nm}$ core taper, a very high sensitivity of $-56.2 \mathrm{pm} / \mathrm{K}$ is obtained. In the untapered case, with the temperature increase, the group birefringence decreases; and since light is very well confined to the core, the dominant contribution comes from the thermo-optic coefficient of silica.

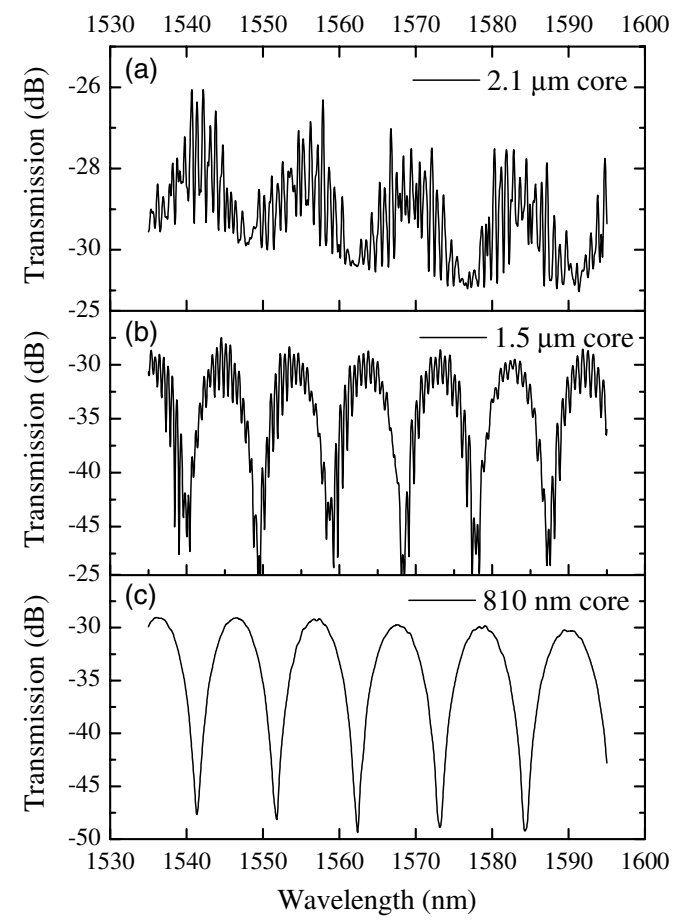

Fig. 6 Spectral response of (a) untapered 2.1- $\mu$ m core fiber; (b) 1.5- $\mu \mathrm{m}$ core taper; and (c) 810-nm core taper in Sagnac interferometer configurations. 


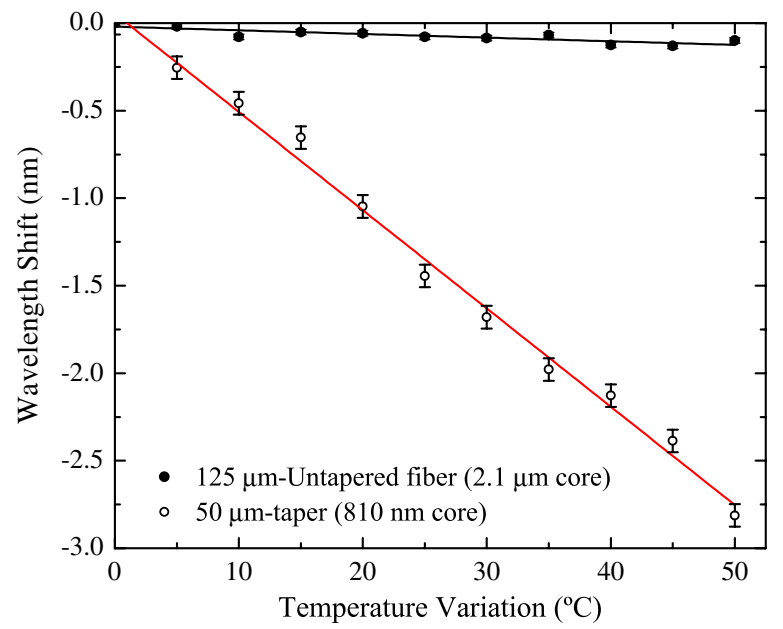

Fig. 7 Wavelength shift with temperature variation for the $2.1-\mu \mathrm{m}$ core and $810-\mathrm{nm}$ core.

In the 810-nm nanowire case, light is less confined to the core and the air starts having a more significant contribution. The large difference in thermo-optic coefficients of silica $\left(8.6 \times 10^{-6} \mathrm{~K}^{-1}\right.$ at $300 \mathrm{~K}$ and $1.5 \mu \mathrm{m}$ wavelength) and air $\left(-9 \times 10^{-7} \mathrm{~K}^{-1}\right)$ is the main reason responsible for the higher sensitivity as this will induce a larger change in birefringence with temperature increase. One mode is much more affected by the air than the other due to different confinements at this scale.

\section{Conclusions}

To summarize, tapers in highly birefringent, triangular core suspended-core fibers were produced by filament heating. Triangular shaped nanowires were produced with core diameters down to $330 \mathrm{~nm}$ with no distortion of the cross-section. Suspended optical nanowires with diameters from $2.1 \mu \mathrm{m}$ to $810 \mathrm{~nm}$ were further analyzed as sensors. The sensors were deemed insensitive to external index variations, which concede them a higher level of protection when compared to conventional optical nanowires. The size-dependent number of guided modes allows for a clean sinusoidal interference pattern through the control of the number of modes. The group birefringence can also be tuned by simply manipulating the taper core dimensions. Finally, the temperature sensitivity can be greatly enhanced from $-2.1 \mathrm{pm} / \mathrm{K}(2.1-\mu \mathrm{m}$ diameter $)$ to $-56.2 \mathrm{pm} / \mathrm{K}$ (810-nm diameter), a 27-fold increase.

When compared with conventional optical nanowires, the suspended nanowires presented show a higher degree of protection, easier handling, and faster connection to standard fiber. Due to their triangular shape, they also present high birefringence, essential for many sensing applications. Interferometers based on this effect can then be used to measure temperature in liquids and gases with high stability of the spectral signals and independence of the external medium.

\section{Acknowledgments}

This work was supported by COST TD1001. This work is financed by the ERDF-European Regional Development Fund through the COMPETE Programme (operational programme for competitiveness) and by National Funds through the FCT-Fundação para a Ciência e a Tecnologia (Portuguese Foundation for Science and Technology) within project (FCOMP01-0124-FEDER-022701). The work of R.M.A. was supported by Fundação para a Ciência e a Tecnologia through the PhD scholarship SFRH/BD/84048/2012. K.S. designed and produced the fiber, M.B. and M.R. fabricated the tapers, R.A. and M.M. characterized and calibrated the sensor, and O.F. and H.B. supervised the work. 


\section{References}

1. L. Tong et al., "Subwavelength-diameter silica wires for low-loss optical wave guiding," Nature 426(6968), 816-819 (2003), http://dx.doi.org/10.1038/nature02193.

2. G. Brambilla, V. Finazzi, and D. J. Richardson, "Ultra-low-loss optical fiber nanotapers," Opt. Express 12(10), 2258-2263 (2004), http://dx.doi.org/10.1364/OPEX.12.002258.

3. G. Brambilla, F. Xu, and X. Feng, "Fabrication of optical fibre nanowires and their optical and mechanical characterisation," Electron. Lett. 42(9), 517-518 (2006), http://dx.doi.org/ 10.1049/el:20060611.

4. G. Brambilla and D. N. Payne, "The ultimate strength of glass silica nanowires," Nano Lett. 9(2), 831-835 (2009), http://dx.doi.org/10.1021/n1803581r.

5. F. Xu, P. Horak, and G. Brambilla, "Optical microfiber coil resonator refractometric sensor," Opt. Express 15(12), 7888-7893 (2007), http://dx.doi.org/10.1364/OE.15 .007888 .

6. T. M. Monro et al., "Sensing with microstructured optical fibres," Meas. Sci. Technol. 12(7), 854-858 (2001), http://dx.doi.org/10.1088/0957-0233/12/7/318.

7. T. M. Monro et al., "Sensing with suspended-core optical fibers," Opt. Fiber Technol. 16(6), 343-356 (2010), http://dx.doi.org/10.1016/j.yofte.2010.09.010.

8. E. C. Magi, P. Steinvurzel, and B. J. Eggleton, "Transverse characterization of tapered photonic crystal fibers," J. Appl. Phys. 96(7), 3976-3982 (2004), http://dx.doi.org/10.1063/1 .1782962 .

9. H. C. Nguyen et al., "Tapered photonic crystal fibres: properties, characterisation and applications," Appl. Phys. B 81(2-3), 377-387 (2005), http://dx.doi.org/10.1007/s00340-0051901-7.

10. Y. K. Lizé et al., "Microstructured optical fiber photonic wires with subwavelength core diameter," Opt. Express 12(14), 3209-3217 (2004), http://dx.doi.org/10.1364/OPEX.12 .003209 .

11. M. Liao et al., "A suspended core nanofiber with unprecedented large diameter ratio of holey region to core," Opt. Express 18(9), 9088-9097 (2010), http://dx.doi.org/10.1364/ OE.18.009088.

12. H. Ebendorff-Heidepriem, S. C. Warren-Smith, and T. M. Monro, "Suspended nanowires: fabrication, design and characterization of fibers with nanoscale cores," Opt. Express 17(4), 2646-2657 (2009), http://dx.doi.org/10.1364/OE.17.002646.

13. A. Hartung, A. M. Heidt, and H. Bartelt, "Pulse-preserving broadband visible supercontinuum generation in all-normal dispersion tapered suspended-core optical fibers," Opt. Express 19(13), 12275-12283 (2011), http://dx.doi.org/10.1364/OE.19 .012275 .

14. O. Frazão, J. P. Carvalho, and H. M. Salgado, "Low-loss splice in a microstructured fibre using a conventional fusion splicer," Microwave Opt. Technol. Lett. 46(2), 172-174 (2005), http://dx.doi.org/10.1002/(ISSN)1098-2760.

15. Y. Liu et al., "High-birefringence fiber loop mirrors and their applications as sensors," Appl. Opt. 44(12), 2382-2390 (2005), http://dx.doi.org/10.1364/AO.44.002382.

16. P. Hlubina, T. Martynkien, and W. Urbanczyk, "Measurements of birefringence dispersion and intermodal dispersion in a two-mode elliptical-core optical fibre using an interferometric method," Opt. Int. J. Light Electron Opt. 115(3), 109-114 (2004), http://dx.doi.org/ 10.1078/0030-4026-00338.

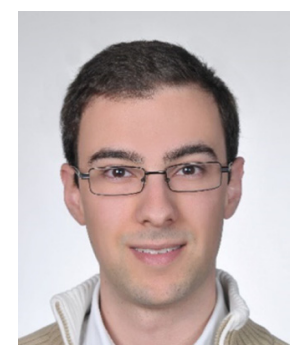

Ricardo M. André received his MSc degree in engineering physics from the University of Porto, Portugal, in 2012. He is currently working toward the doctoral degree in physics from the University of Porto in collaboration with INESC Porto, Portugal. His current interests include optical fiber sensors, fabrication of micro- and nanodevices in optical fiber and postprocessing of optical fiber for sensing applications. Currently, in the scope of this $\mathrm{PhD}$ thesis, he is working on the development of new fibers and fiber configurations for further postprocessing with focused ion beam, chemical etching, and femtosecond laser. 

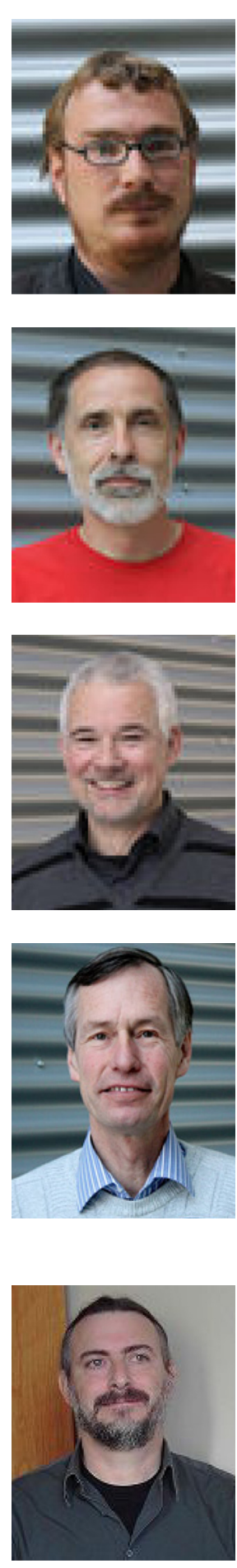

Manuel B. Marques received a degree in physics from Porto University in 1983, and a PhD degree in physics from the same university in 1991. His thesis work was on nonlinear coupling to optical waveguides. From 1993 to 2001, he was a member of Centro de Física do Porto, where he worked on fiber lasers and integrated optics. He joined INESC Porto, Portugal, in 2002, where he has worked on fiber lasers and sensors. He has been an assistant professor in the Physics Department of Porto University since 1991.

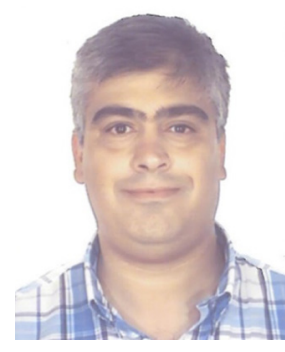

Orlando Frazão graduated in physics engineering (optoelectronics and electronics) from the University of Aveiro, Aveiro, Portugal. He received his $\mathrm{PhD}$ from University of Porto, Porto, Portugal, on optical fiber sensors based on interferometry and nonlinear effects. From 1997 to 1998, he was with the Institute of Telecommunications, Aveiro, with a scholarship in the European Project UPGRADE (High Bitrate $1300 \mathrm{~nm}$ Upgrade of the European Standard Single Mode Network). Presently, he is a senior researcher at the Optoelectronics and Electronic Systems Unit at INESC Porto and leads the group of optical fiber sensors for physical measurement. His present research interests include optical fiber sensors and optical communications. 